\title{
Sabit Fuzzy Nokta Teoremleri
}

Fixed Fuzzy Point Theorems

\author{
Ferhan ŞOLA ERDURAN*
}

Gazi Üniversitesi, Fen Fakültesi, Matematik Bölümü, 06500, Ankara

• Geliş tarihi / Received: 19.09.2019 • Düzeltilerek geliş tarihi / Received in revised form: 05.05.2020 • Kabul tarihi / Accepted: 13.05 .2020

\section{Öz}

$\mathrm{Bu}$ çalışmada, fuzzy alt kümeler ailesi üzerinde verilmiş olan Hausdorrf fuzzy metrik uzaylarda, uzaklığ fonksiyonlar yardımıyla ilk olarak fuzzy fonksiyonları için sabit fuzzy nokta teoremi ispatlanmış ve teorem örneklerle desteklenmiştir. Daha sonra, ana teoremin bir uygulaması olarak ortak sabit fuzzy nokta teoremi ve ispatı verilmiştir.

Anahtar kelimeler: Fuzzy Metrik, Sabit Fuzzy Nokta, Uzaklığı Değiștiren Fonksiyon

\begin{abstract}
In this study, firstly, fuzzy fixed-point theorem was proved for fuzzy mappings by altering distance functions in Hausdorff fuzzy metric spaces which are given on family of fuzzy subsets and the theorem was supported by examples. After that, common fuzzy fixed-point theorem and its proof were given as an application of main theorem.
\end{abstract}

Keywords: Fuzzy Metric, Fixed Fuzzy Point, Altering Distance Function 


\section{Giriş}

Günlük yaşantımızda doğruluğu kişiden kişiye değişen pek çok durumla karşılaşmaktayız. Örneğin zeki öğrenci, yaşlı kadın, ılık su, pahalı ev denildiğinde ortaya çıkan belirsizliğin matematiksel olarak tanımlanması oldukça zordur. Bunun için interval matematik, olasılık teori, fuzzy küme teori gibi teoriler geliştirilerek belirsizlik, modern anlamda matematiksel olarak modellenmeye çalışılmıştır. $\mathrm{Bu}$ teoriler arasında belirsizlikleri doğru çözüme kavuşturma konusunda ortaya konulan en uygun teori, Zadeh (1965) tarafindan verilen fuzzy (bulanık) kümeler teorisidir. Fuzzy küme, doğru veya yanlış, 0 veya 1 ikili mantık sistemi yerine doğruluk değeri $[0,1]$ aralığında değişen dereceli değerler almaktadır. Böylece, klasik her küme bir fuzzy küme olarak ifade edilebilmektedir.

Zadeh'in makalesinden sonra (Chang, 1968) ve (Lowen, 1976) fuzzy topolojik uzay kavramını tanımlayarak klasik topolojide yapılan çalışmalar için yeni bir alan oluşturmuşlardır. (Kramosil ve Michalek, 1975) fuzzy metrik tanımından sonra Hausdorff uzay yapısının elde edilebilmesi için (George ve Veeramani, 1994) tarafından fuzzy metrik uzay tanımı tekrar verilmiş ve bu sayede klasik analizde yapılan pek çok çalışma bu uzaylara taşınmıştır.

Fuzzy metrik uzaylar, özellikle sabit nokta teori araştırmacıları tarafından yoğun ilgi görmüş, farklı koşullar altında bir fonksiyonun sabit noktasının varlığ 1 ve tekliği incelenmiştir. (Chitra ve Subrahmanyam, 1987; Mihet, 2004; Türkoğlu vd., 2006; Alaca, 2009). (Heilpern, 1981) fuzzy fonksiyon kavramını vermiş ve bu fonksiyonların sabit noktalarını bulmada ilk kez Hausdorff metrik uzayı, (Nadler, 1969) in klasik metrik uzaylarda çoğul değerli fonksiyonlar için verdiği sabit nokta teoreminden yola çıkarak kullanmıştır. (Rodriguez-Lopez ve Romaguera, 2004) ise bir fuzzy metrik uzayın boş olmayan kompakt alt kümelerinin ailesi üzerinde Hausdorff fuzzy metriği tanımlamış ve bu metriğin tamlık, prekompaktlık ve tamlama gibi çeşitli özelliklerini incelemişlerdir.

Yakın zamanda (Phiangsungnoen vd., 2014) bir fuzzy metrik uzayın fuzzy alt kümelerinin boştan farklı ve kompakt seviye kümelerinin ailesi üzerinde Hausdorff fuzzy metriği kullanarak ilgi çekici sabit fuzzy nokta teoremleri ispatlamışlardır. Hemen ardından (Abbas vd., 2015), Hausdorff fuzzy metrik uzaylarda genelleştirilmiş büzülme şartını sağlayan fonksiyonların sabit ve ortak sabit fuzzy noktalarını bulmaya yarayan daha genel sonuçlar elde etmişlerdir.

Diğer taraftan, (Khan vd., 1984) klasik metrik uzaylardaki sabit nokta teori çalışmalarında kullandıkları ve uzaklığı değiştiren fonksiyon olarak adlandırdıkları fonksiyon yardımıyla aşağıdaki sonucu elde etmişlerdir:

\section{Tanım 1.1}

$\phi:[0, \infty) \rightarrow[0, \infty)$ dönüşümü;

i) $\phi(t)=0 \Leftrightarrow t=0$,

ii) sürekli ve monoton azalmayan

özelliklerini sağlarsa uzaklığı değiştiren fonksiyon olarak tanımlanır (Khan vd., 1984).

\section{Teorem 1.2}

$(X, d)$ tam metrik uzay, $\phi$ uzaklığı değiştiren fonksiyon olmak üzere her $x, y \in X, 0<c<1$ için $f: X \rightarrow X$ fonksiyonu

$\phi(d(f x, f y)) \leq c \phi(d(x, y))$

eşitsizliğini sağlasın. Bu durumda $f$ nin bir tek sabit noktası vardır (Khan vd., 1984).

Gerek klasik metrik gerekse fuzzy metrik uzaylarda, bazı özel koşullar eklenerek uzaklığı değiştiren fonksiyon yardımıyla pek çok sabit nokta teori çalışmaları yapılmıştır (Naidu, 2003; Popa ve Mocanu,2009; Shen vd., 2012; Nashine ve Aydi, 2013; Dosenovic vd., 2014).

Daha sonra (Rhoades, 2001) tanım olarak her ne kadar uzaklığg değiştiren fonksiyona benzese de kullanım şekli tamamen farklı olan zayıf büzülme dönüşümünü kullanarak aşağıdaki sonucu elde etmiştir:

Tanım 1.3

$(X, d)$ metrik uzay, $\varphi:[0, \infty) \rightarrow[0, \infty), \varphi(t)=$ $0 \Leftrightarrow t=0$ özelliğini sağlayan artan sürekli bir fonksiyon olmak üzere $T: X \rightarrow X$ dönüşümü, her $x, y \in X$ için

$d(T x, T y) \leq d(x, y)-\varphi(d(x, y))$

eşitsizliğini sağlar ise $T$ ye zayıf büzülme denir (Rhoades, 2001).

\section{Teorem 1.4}

$(X, d)$ metrik uzay olsun. $T: X \rightarrow X$ zayıf büzülme dönüşümü ise $T$ nin bir tek sabit noktası vardır (Rhoades, 2001). 
Biz de bu çalışmada, fuzzy alt kümeler ailesi üzerinde uyarlanmış olan Hausdorff fuzzy metrik uzaylarda uzaklığı değiştiren fonksiyonlar vasitasıyla sabit fuzzy nokta ve ortak sabit fuzzy nokta teoremleri ile ana teoremi destekleyen örnekler verdik.

\section{Temel Bilgiler}

$\mathrm{Bu}$ bölümde ileride kullanılacak olan bazı tanım ve örnekler verilmiştir.

\subsection{Tanım}

$X \neq \emptyset$ herhangi bir küme ve $I=[0,1] \subset \mathbb{R}$ olsun. $\mu_{A}: X \rightarrow[0,1]$ fonksiyonu tarafindan karakterize edilen $A=\left\{\left(x, \mu_{A}(x)\right): x \in X\right\} \subset X \times I$ kümesine $X$ de bir fuzzy küme denir. Burada $\mu_{A}$ ya $A$ fuzzy kümesinin üyelik fonksiyonu ve her $x \in X$ için $\mu_{A}(x) \in I$ değerine de $x$ in $A$ ya ait olma derecesi denir. $X$ den $I$ ya tanımlanan bütün fonksiyonların kümesi $I^{X}$ ile gösterilir ve her bir elemanı bir fuzzy kümesidir (Zadeh, 1965).

Buradan itibaren yazım kolaylığı açısından $\mu_{A}(x)$ yerine $A(x)$ gösterimi kullanılacaktır.

\subsection{Tanım}

$X \neq \emptyset, \quad A \in I^{X} \quad$ ve $\quad \alpha \in(0,1] \quad$ olsun. $[A]_{\alpha}=$ $\{x \in X: A(x) \geq \alpha\}$ kümesine $A$ nın $\alpha$ seviye kümesi denir. Ĕger, $X$ kümesi üzerinde bir topoloji varsa, $\bar{B}, B$ nin $X$ deki topolojiye göre kapanışı olmak üzere

$$
[A]_{0}=\overline{\{x \in X: A(x)>0\}}
$$

dır. Ayrıca $0<\alpha<\beta \leq 1$ için, $[A]_{\beta} \subset[A]_{\alpha}$ dır (Wong, 1974).

\subsection{Tanım}

Her $\alpha \in(0,1]$ ve $x \in X$ için üyelik fonksiyonu

$$
x_{\alpha}(y)=\left\{\begin{array}{lc}
\alpha, & y=x \\
0, & \text { diğger durumda }
\end{array}\right.
$$

şeklinde tanımlanan $x_{\alpha}: X \rightarrow[0,1]$ fuzzy kümesine $X$ de bir fuzzy nokta denir (Wong, 1974).

\subsection{Tanim}

$X \neq \varnothing$ bir küme, $A \in I^{X}$ ve $x_{\alpha}$ bir fuzzy nokta olsun. $\forall x \in X$ için $\alpha \leq A(x)$ ise, $x_{\alpha} \in A$ denir. Bir fuzzy küme, bu fuzzy kümeye ait fuzzy noktaların birleşimi şeklinde yazılabilir, dolayısıyla $A$ ya ait her fuzzy nokta aynı zamanda $A$ nın fuzzy alt kümesidir (Pao-Ming ve YingMing, 1980).

\subsection{Tanim}

$*:[0,1] \times[0,1] \rightarrow[0,1]$ ikili işlemi, $\forall a, b, c, d \in$ $[0,1]$ için,

i) * değişmeli ve birleşmeli,

ii) * sürekli,

iii) $a * 1=a$,

iv) $a \leq c$ ve $b \leq d$ iken $a * b \leq c * d$

şartlarını sağlıyorsa, * ikili işlemine sürekli üçgen norm (sürekli $t$-norm) denir (Schweizer ve Sklar, 1960).

\section{6. Örnek}

$\forall a, b \in[0,1]$ için, $a * b=\min \{a, b\}, a * b=a b$, $a * b=$ maks $\{a+b-1,0\}$ şeklinde tanımlanan ikili işlemler sürekli $t$-normdur.

\subsection{Tanım}

$X \neq \emptyset$ herhangi bir küme, $*$ işlemi sürekli $t$-norm $M: X \times X \times(0, \infty) \rightarrow(0,1]$ fonksiyonu; $\forall x, y, z \in$ $X$ ve $s, t>0$ için,

FM-1) $M(x, y, t)>0$,

FM-2) $M(x, y, t)=1 \Leftrightarrow x=y$,

FM-3) $M(x, y, t)=M(y, x, t)$

FM-4) $M(x, y, t) * M(y, z, s) \leq M(x, z, t+s)$,

FM-5) $M(x, y, \cdot):(0, \infty) \rightarrow(0,1]$ sürekli

koşullarını sağlarsa $M$ ye fuzzy metrik, $(X, M, *)$ üçlüsüne de fuzzy metrik uzay denir (George ve Veeramani, 1994).

\section{8. Örnek}

$X \neq \emptyset$ herhangi bir küme, $f: X \rightarrow \mathbb{R}^{+}$bire bir fonksiyon ve $g: \mathbb{R}^{+} \rightarrow[0, \infty)$ artan sürekli fonksiyon olsun. $\alpha, \beta>0$ sabit say1lar ve $a * b=$ $a b$ olmak üzere her $x, y \in X$ ve $t>0$ için,

$M(x, y, t)=\left(\frac{(\min \{f(x), f(y)\})^{\alpha}+g(t)}{(\operatorname{maks}\{f(x), f(y)\})^{\alpha}+g(t)}\right)^{\beta}$

şeklinde tanımlanan $M$ fonksiyonu, $X$ üzerinde bir fuzzy metriktir.

Eğer, $f=I$ (birim fonksiyon) ve $\alpha=\beta=1$ olarak alınırsa,

a) $X=\mathbb{R}^{+}$ve $g=I$ olması halinde 
$M(x, y, t)=\frac{\min \{x, y\}+t}{\operatorname{maks}\{x, y\}+t}$

b) $X=\mathbb{N}$ ve $g(t)=0$ olması halinde

$M(x, y, t)=\frac{\min \{x, y\}}{\operatorname{maks}\{x, y\}}$

fuzzy metrikleri elde edilir (Gregori vd., 2011).

\section{9. Örnek}

$(X, d)$ bir metrik uzay, her $a, b \in[0,1]$ için $a *$ $b=a b$ (veya $a * b=\min \{a, b\})$ olmak üzere her $x, y \in X$ ve $t>0$ için,

$M(x, y, t)=\frac{t}{t+d(x, y)}$

şeklinde tanımlanan $M$ fonksiyonu, $X$ üzerinde bir fuzzy metriktir. Bu fuzzy metriğe, $d$ metriğinden indirgenen standart fuzzy metrik denir (George ve Veeramani, 1994).

\subsection{Tanım}

$(X, M, *)$ bir fuzzy metrik uzay, $\varnothing \neq B \subset X$ ve $a \in$ $X$ olsun.

$M(a, B, t)=\sup \{M(a, b, t): b \in B\}$

ifadesine $a$ nın $t$ ye göre $B$ kümesine yakın olma derecesi denir (Veeramani, 2001).

\subsection{Tanım}

$(X, M, *)$ bir fuzzy metrik uzay ve $\left\{x_{n}\right\} \subset X$ olsun. $\forall t>0$ için $\lim _{n \rightarrow \infty} M\left(x_{n}, x, t\right)=1$ şartı sağlanırsa $\left\{x_{n}\right\}$ dizisi $x$ noktasına yakınsar denir ve $\lim _{n \rightarrow \infty} x_{n}=x \quad$ şeklinde gösterilir (George ve Veeramani,1994).

\subsection{Tanım}

$(X, M, *)$ bir fuzzy metrik uzay ve $\left\{x_{n}\right\} \subset X$ olsun.

i) Her $t>0$ ve her $p \in \mathbb{N}$ için, $\lim _{n \rightarrow \infty} M\left(x_{n+p}, x_{n}, t\right)=1$ ise $\left\{x_{n}\right\}$ dizisine GCauchy dizisi denir.

ii) $X$ deki her G-Cauchy dizisi yakınsak ise, $(X, M, *)$ fuzzy metrik uzayına G-tamdır denir (Grabiec, 1988).

\subsection{Tanım}

$(X, M, *)$ bir fuzzy metrik uzay ve $A \subset X$ olsun.

i) $A$ içindeki yakınsak her dizinin limiti $A$ da ise, yani $\left\{x_{n}\right\} \subset A$ ve $x_{n} \rightarrow x$ iken $x \in A$ ise, $A$ ya $X$ de kapalıdır denir. ii) $A$ içindeki her dizinin $A$ da en az bir yakınsak alt dizisi var ise, $A$ kümesine $X$ de kompakt denir (George ve Veeramani, 1994).

\section{Hausdorff Fuzzy Metrik Uzaylar}

\subsection{Tanım}

$(X, M, *)$ bir fuzzy metrik uzay ve $K(X), X$ in boştan farklı kompakt alt kümelerinin ailesini göstermek üzere her $A, B \in K(X)$ ve $t>0$ için,

$H_{M}(A, B, t)=$
$\min \left\{\inf _{a \in A} M(a, B, t), \inf _{b \in B} M(A, b, t)\right\}$

ile tanımlanan $H_{M}: K(X) \times K(X) \times(0, \infty) \rightarrow$ $[0,1]$ fonksiyonuna $M$ fuzzy metriğinin oluşturduğu Hausdorff fuzzy metrik denir (Rodriguez-Lopez ve Romaguera, 2004).

\subsection{Tanim}

$(X, M, *)$ bir fuzzy metrik uzay olmak üzere $X$ deki $\alpha$ seviye kümeleri boştan farklı ve kompakt olan fuzzy kümelerin ailesini $W_{\alpha}(X)$ ile gösterelim. Yani,

$W_{\alpha}(X)=\left\{A \in I^{X}:[A]_{\alpha} \neq \emptyset\right.$ ve kompakt $\}$.

$A, B \in W_{\alpha}(X)$ ve $\alpha \in[0,1]$ için,

$M_{\alpha}(x, B, t)=\sup _{y \in[B]_{\alpha}} M(x, y, t)$

şeklinde tanımlanan $M_{\alpha}, \alpha$ nın artmayan bir fonksiyonu ve

$H_{M_{\alpha}}(A, B, t)=H_{M}\left([A]_{\alpha},[B]_{\alpha}, t\right)$

şeklinde tanımlanan $H_{M_{\alpha}}$ da $W_{\alpha}(X)$ üzerinde $M$ fuzzy metriğinin oluşturduğu Hausdorff fuzzy metriktir (Abbas vd., 2015).

\section{3. Önerme}

$(X, M, *)$ bir fuzzy metrik uzay, $x, y \in X$ ve $A, B \in$ $W_{\alpha}(X)$ olmak üzere aşağıdaki ifadeler sağlanır:

a) Her $x \in X$ ve $t>0$ için $M_{\alpha}(x, B, t)=$ $M\left(x, b_{0}, t\right)$ olacak şekilde en az bir $\left(b_{0}\right)_{\alpha} \subset B$ vardir.

b) $M_{\alpha}(x, A, t)=1$ ise, $x_{\alpha} \subset A$ dir.

c) $M\left(x, y_{x}, t\right)=M_{\alpha}(x, B, t)$ olacak şekildeki $\left(y_{x}\right)_{\alpha} \subset B \quad$ için $\quad M_{\alpha}(x, C, t+s) \geq$ $M_{\alpha}(x, B, t) * M_{\alpha}\left(y_{x}, C, s\right)$ dir (Abbas vd., 2015). 


\subsection{Tanım}

$(X, M, *)$ bir fuzzy metrik uzay, $Y \subset X$ herhangi bir alt küme olmak üzere $F: Y \rightarrow W_{\alpha}(X)$ fonksiyonuna $Y$ üzerinde fuzzy fonksiyonu denir. Her $y \in Y$ için $F y: X \rightarrow[0,1]$ üyelik fonksiyonu tarafindan karakterize edilen $F y$ kümesi $X$ de bir fuzzy kümesidir. $F y(x), x \in X$ in $F y$ fuzzy kümesine aitlik derecesi olup, $Y$ üzerindeki $F$ fuzzy fonksiyonu, $Y \times X$ in bir fuzzy alt kümesidir (Abbas vd., 2015).

\subsection{Tanım}

$X \neq \emptyset$ herhangi bir küme ve $F, X$ üzerinde fuzzy fonksiyonu olsun. $x_{\alpha} \subset F x$, yani $\alpha(x) \leq F x(x)$ ya da $x \in[F x]_{\alpha}$ ise, $x_{\alpha}$ ya $F$ fuzzy fonksiyonunun sabit fuzzy noktas 1 denir. Burada $x$ in $F x$ deki sabitlik derecesi en az $\alpha$ dır. Eğer, $x_{1} \subset F x$ ise, $x$ noktasina $F$ fuzzy fonksiyonunun sabit noktas1 denir (Estruch ve Vidal, 2001).

\subsection{Tanım}

$(X, M, *)$ fuzzy metrik uzay, $F: X \times X \rightarrow W_{\alpha}(X)$ bir fuzzy fonksiyonu ve $g: X \rightarrow X$ bir fonksiyon olmak üzere

a) $(g x)_{\alpha} \subset F x$, yani $g x \in[F x]_{\alpha}$ ise, $x_{\alpha}$ ya $g$ ile $F$ nin çakışı sabit fuzzy noktası denir. $g$ ile $F$ nin tüm çakışık sabit fuzzy noktalarının kümesi $C_{\alpha}(g, F)$ ile gösterilir.

b) Eğer $x_{\alpha}=(g x)_{\alpha} \subset F x$, yani $x=g x \in[F x]_{\alpha}$ ise, $x_{\alpha}$ ya $g$ ile $F$ nin ortak sabit fuzzy noktasi denir (Ali ve Abbas, 2013).

\section{Sabit Fuzzy Nokta Teoremleri}

$\mathrm{Bu}$ bölümde, uzaklığı değiş̧iren fonksiyonlar vasıtasıyla fuzzy fonksiyonları için sabit ve ortak sabit nokta teoremleri verilmiştir.

\section{1. Önerme}

$X \neq \varnothing$ ve $g: X \rightarrow X$ bir fonksiyon olsun. Bu durumda $g(E)=g(X)$ ve $g: E \rightarrow X$ bire bir olacak şekilde bir $E \subset X$ vardır (Haghi vd., 2011).

\subsection{Teorem}

$(X, M, *)$ G-tam fuzzy metrik uzay, $\phi$ uzaklığı değiştiren fonksiyon ve $\psi:[0, \infty) \rightarrow[0, \infty)$, $\psi(t)=0 \Leftrightarrow t=0$ özelliğini sağlayan alt yarı sürekli bir fonksiyon olmak üzere her $x, y \in X$ ve her $t>0$ için $F: X \rightarrow W_{\alpha}(X)$ fuzzy fonksiyonu;

$\phi\left(\frac{1}{H_{M_{\alpha}}\left(F x_{n}, F x, t\right)}-1\right) \leq \phi\left(\frac{1}{M\left(x_{n}, x, t\right)}-1\right)-$

$\psi\left(\frac{1}{M\left(x_{n}, x, t\right)}-1\right)$

şartını sağlasın. Bu durumda $F$ nin sabit fuzzy noktasi vardır.

\section{İspat}

$x_{0} \in X$ verilsin. $\left[F x_{0}\right]_{\alpha} \neq \varnothing$ olduğundan $x_{1} \in$ $\left[F x_{0}\right]_{\alpha}$ olacak şekilde $x_{1} \in X$ vardır. $\left[F x_{1}\right]_{\alpha} \neq \varnothing$ ve kompakt olduğundan Önerme 3.3 den $x_{2} \in$ $\left[F x_{1}\right]_{\alpha}$ var, öyle ki

$$
\begin{aligned}
\phi\left(\frac{1}{M\left(x_{1}, x_{2}, t\right)}-1\right) & =\phi\left(\frac{1}{\sup _{y \in\left[F x_{1}\right]_{\alpha}} M\left(x_{1}, y, t\right)}-1\right) \\
& \leq \phi\left(\frac{1}{H_{M_{\alpha}}\left(F x_{0}, F x_{1}, t\right)}-1\right) \\
& \leq \phi\left(\frac{1}{M\left(x_{0}, x_{1}, t\right)}-1\right)-\psi\left(\frac{1}{M\left(x_{0}, x_{1}, t\right)}-1\right) \\
& <\phi\left(\frac{1}{M\left(x_{0}, x_{1}, t\right)}-1\right)
\end{aligned}
$$

olup $\phi$ azalmayan olduğundan $M\left(x_{1}, x_{2}, t\right)>M\left(x_{0}, x_{1}, t\right)$ bulunur.

$\mathrm{Bu}$ şekilde devam edilerek $\left[F x_{n}\right]_{\alpha} \neq \emptyset$ ve kompakt olduğundan Önerme 3.3 den $x_{n+1} \in\left[F x_{n}\right]_{\alpha}$ var, öyle ki

$$
\phi\left(\frac{1}{M\left(x_{n}, x_{n+1}, t\right)}-1\right)=\phi\left(\frac{1}{\sup _{y \in\left[F x_{n}\right]_{\alpha}} M\left(x_{n}, y, t\right)}-1\right)
$$




$$
\begin{aligned}
& \leq \phi\left(\frac{1}{H_{M_{\alpha}}\left(F x_{n-1}, F x_{n}, t\right)}-1\right) \\
& \leq \phi\left(\frac{1}{M\left(x_{n-1}, x_{n}, t\right)}-1\right)-\psi\left(\frac{1}{M\left(x_{n-1}, x_{n}, t\right)}-1\right) \\
& <\phi\left(\frac{1}{M\left(x_{n-1}, x_{n}, t\right)}-1\right)
\end{aligned}
$$

olup $\phi$ azalmayan olduğundan $M\left(x_{n}, x_{n+1}, t\right)>M\left(x_{n-1}, x_{n}, t\right)$ bulunur.

O halde her $n \in \mathbb{N}$ için $M\left(x_{n}, x_{n+1}, t\right)>M\left(x_{n-1}, x_{n}, t\right)$ olup dolayısıyla $(0,1]$ aralığında pozitif reel sayıların artan $\operatorname{bir}\left\{M\left(x_{n-1}, x_{n}, t\right)\right\}$ dizisi elde edilmiş olur. $\lim _{n \rightarrow \infty} M\left(x_{n-1}, x_{n}, t\right)=\delta(t)$ diyelim ve $\delta(t)=1$ olduğunu gösterelim. Kabul edelim ki $\delta(t)<1$ olacak şekilde $t>0$ var olsun. Yukarıdaki eşitsizlikte $n \rightarrow$ $\infty$ iken limit alınırsa,

$\phi\left(\frac{1}{\delta(t)}-1\right) \leq \phi\left(\frac{1}{\delta(t)}-1\right)-\psi\left(\frac{1}{\delta(t)}-1\right)<\phi\left(\frac{1}{\delta(t)}-1\right)$

olur ki bu bir çelişkidir. O halde $\lim _{n \rightarrow \infty} M\left(x_{n-1}, x_{n}, t\right)=1$ dir. Her $p \in \mathbb{N}$ için

$M\left(x_{n}, x_{n+p}, t\right) \geq M\left(x_{n}, x_{n+1}, t / p\right) * M\left(x_{n+1}, x_{n+2}, t / p\right) * \ldots * M\left(x_{n+p-1}, x_{n+p}, t / p\right)$

ve böylece $\lim _{n \rightarrow \infty} M\left(x_{n}, x_{n+p}, t\right)=1$ elde edilir. Bu da $\left\{x_{n}\right\}$ dizisinin G-Cauchy dizisi olması demektir. $X$, Gtam fuzzy metrik uzay olduğundan $\lim _{n \rightarrow \infty} x_{n}=x$ olacak şekilde $x \in X$ vardır.

Şimdi de $x \in[F x]_{\alpha}$ olduğunu gösterelim. (14) eşitsizliğinde $x=x_{n}, y=x$ yazılırsa,

$\phi\left(\frac{1}{H_{M_{\alpha}}\left(F x_{n}, F x, t\right)}-1\right) \leq \phi\left(\frac{1}{M\left(x_{n}, x, t\right)}-1\right)-\psi\left(\frac{1}{M\left(x_{n}, x, t\right)}-1\right)$

olup $n \rightarrow \infty$ iken limit alınırsa

$\lim _{n \rightarrow \infty} H_{M_{\alpha}}\left(F x_{n}, F x, t\right)=1$

bulunur. $x_{n+1} \in\left[F x_{n}\right]_{\alpha}$ olduğundan

$\lim _{n \rightarrow \infty} \sup _{y \in[F x]_{\alpha}} M\left(x_{n+1}, y, t\right)=1$

ve dolayısıyla $\lim _{n \rightarrow \infty} M\left(x_{n}, y_{n}, t\right)=1$ olacak şekilde $[F x]_{\alpha}$ da $\left\{y_{n}\right\}$ dizisi elde edilir. Her $n \in \mathbb{N}$ için

$M\left(y_{n}, x, t\right) \geq M\left(y_{n}, x_{n}, \frac{t}{2}\right) * M\left(x_{n}, x, \frac{t}{2}\right)$

yazılır ve $n \rightarrow \infty$ iken limit alınırsa $\lim _{n \rightarrow \infty} M\left(y_{n}, x, t\right)=1$ yani $\lim _{n \rightarrow \infty} y_{n}=x$ bulunur. $[F x]_{\alpha}$ kompakt ve $y_{n} \in$ $[F x]_{\alpha}$ olduğundan $x \in[F x]_{\alpha}$ dir.

Sonuç olarak $x_{\alpha}$ noktası $F$ nin sabit fuzzy noktasıdır.

\section{3. Örnek}

$X=\{1,2,3\}$ ve $d: X \times X \rightarrow \mathbb{R}$ fonksiyonu; her $x \in X$ için

$d(x, x)=0, d(1,2)=9, d(1,3)=12, d(2,3)=15$ olsun. Her $a, b \in[0,1]$ için $a * b=a b$ ve her $x, y \in X$, $t>0$ için $M(x, y, t)=\frac{t}{t+d(x, y)}$ olarak tanımlansın.

$\alpha=\frac{2}{3}$ olmak üzere $F: X \rightarrow W_{\alpha}(X)$ fuzzy fonksiyonunu aşağıdaki şekilde verilsin: 
$(F 1)(x)=\left\{\begin{array}{cc}2 / 3, & x=1 \\ 1 / 3, & x=2 \\ 0, & x=3\end{array} \quad(F 2)(x)=\left\{\begin{array}{cc}3 / 4, & x=1 \\ 0, & x=2 \\ 1 / 2, & x=3\end{array} \quad(F 3)(x)=\left\{\begin{array}{cc}1 / 2, & x=1 \\ 2 / 3, & x=2 \\ 1 / 3, & x=3\end{array}\right.\right.\right.$

$[F 1]_{\alpha}=\{x \in X:(F 1)(x) \geq \alpha\}=\{1\}$ olarak bulunur. Benzer şekilde $[F 2]_{\alpha}=\{1\}$ ve $[F 3]_{\alpha}=\{2\}$ elde edilir. $\phi(x)=x, \psi(x)=\frac{x}{36}$ olmak üzere (14) eşitsizliğinin sağlandığını gösterelim:

1. durum: $x, y \in\{1,2\}$ ise; $H_{M_{\alpha}}(F x, F y, t)=H_{M}(\{1\},\{1\}, t)=1$ olacağından (14) sağlanır.

2. durum: $x=1, y=3$ ise;

$$
\begin{aligned}
H_{M_{\alpha}}(F x, F y, t) & =H_{M}\left([F 1]_{\alpha},[F 3]_{\alpha}, t\right) \\
& =\min \left\{\inf _{x \in[F 1]_{\alpha}} M\left(x,[F 3]_{\alpha}, t\right), \inf _{y \in[F 3]_{\alpha}} M\left([F 1]_{\alpha}, y, t\right)\right\} \\
& =\frac{t}{t+9}
\end{aligned}
$$

olup,

$\phi\left(\frac{1}{H_{M_{\alpha}}(F x, F y, t)}-1\right)=\phi\left(\frac{9}{t}\right)=\frac{9}{t} \leq \frac{35}{3 t}=\frac{12}{t}-\frac{1}{3 t}=\phi\left(\frac{1}{M(x, y, t)}-1\right)-\psi\left(\frac{1}{M(x, y, t)}-1\right)$

yani (14) sağlanır.

3. durum: $x=2, y=3$ ise;

$$
\begin{aligned}
H_{M_{\alpha}}(F x, F y, t) & =H_{M}\left([F 2]_{\alpha},[F 3]_{\alpha}, t\right) \\
& =\min \left\{\inf _{x \in[F 2]_{\alpha}} M\left(x,[F 3]_{\alpha}, t\right), \inf _{y \in[F 3]_{\alpha}} M\left([F 2]_{\alpha}, y, t\right)\right\} \\
& =\frac{t}{t+9}
\end{aligned}
$$

olup,

$\phi\left(\frac{1}{H_{M_{\alpha}}(F x, F y, t)}-1\right)=\phi\left(\frac{9}{t}\right)=\frac{9}{t} \leq \frac{175}{12 t}=\frac{15}{t}-12 \frac{5}{t}=\phi\left(\frac{1}{M(x, y, t)}-1\right)-\psi\left(\frac{1}{M(x, y, t)}-1\right)$

yani (14) sağlanır.

Böylece Teorem 4.2 nin tüm koşulları sağlanmış olur. Ayrıca $1 \in[F 1]_{\alpha}=\{1\}$ olup $x=1, F$ nin sabit noktasidir.

\section{4. Örnek}

$X=[0,1]$ olsun. Her $x, y \in X, t>0$ için $M(x, y, t)=\frac{t}{t+|x-y|}$ ve her $a, b \in[0,1]$ için $a * b=\min \{a, b\}$ olarak tanımlansın. $\alpha \in(0,1]$ olmak üzere $F: X \rightarrow W_{\alpha}(X)$ fuzzy fonksiyonunu;

$F 0(x)=\left\{\begin{array}{cc}\alpha, & x=0 \\ \alpha / 3, & 0<x \leq 1 / 50 \\ 0, & 1 / 50<x \leq 1\end{array}\right.$

ve $r \neq 0$ için,

$\operatorname{Fr}(x)=\left\{\begin{array}{cc}\alpha, & 0 \leq x \leq r / 75 \\ 2 \alpha / 3, & r / 75<x \leq r / 15 \\ \alpha / 4, & r / 15<x \leq 1\end{array}\right.$ 
şeklinde verilsin. $\phi, \psi$ fonksiyonları Örnek 4.3 deki gibi alınsın. Buna göre;

1. durum: $x=y=0$ ise, $H_{M_{\alpha}}(F x, F y, t)=H_{M}(\{0\},\{0\}, t)=1$ olacağından (14) sağlanır.

2. durum: $x=0$ ve $y \neq 0$ ise, $[F x]_{\alpha}=\{0\}$ ve $[F y]_{\alpha}=[0, y / 75]$ olarak bulunur. Buradan,

$$
\begin{aligned}
H_{M_{\alpha}}(F x, F y, t) & =H_{M}\left([F x]_{\alpha},[F y]_{\alpha}, t\right) \\
& =H_{M}(\{0\},[0, y / 75], t) \\
& =\frac{t}{t+y / 75}
\end{aligned}
$$

olup,

$\phi\left(\frac{1}{H_{M_{\alpha}}(F x, F y, t)}-1\right)=\phi\left(\frac{y}{75 t}\right)=\frac{y}{75 t} \leq \frac{35 y}{36 t}=\phi\left(\frac{1}{M(x, y, t)}-1\right)-\psi\left(\frac{1}{M(x, y, t)}-1\right)$.

3. durum: $x \neq 0$ ve $y \neq 0$ ise, $[F x]_{\alpha}=[0, x / 75]$ ve $[F y]_{\alpha}=[0, y / 75]$ olarak bulunur. Öyleyse,

$$
\begin{aligned}
H_{M_{\alpha}}(F x, F y, t) & =H_{M}\left([F x]_{\alpha},[F y]_{\alpha}, t\right) \\
& =H_{M}([0, x / 75],[0, y / 75], t) \\
& =\frac{75 t}{75 t+|x-y|}
\end{aligned}
$$

olup,

$\phi\left(\frac{1}{H_{M_{\alpha}}(F x, F y, t)}-1\right)=\phi\left(\frac{|x-y|}{75 t}\right)=\frac{|x-y|}{75 t} \leq \frac{35|x-y|}{36 t}=\phi\left(\frac{1}{M(x, y, t)}-1\right)-\psi\left(\frac{1}{M(x, y, t)}-1\right)$.

Dolayısıyla, Teorem 4.2 nin tüm koşulları sağlanmış olur. Ayrıca $0 \in[F 0]_{\alpha}=\{0\}$ olup $x=0, F$ nin sabit noktasidir.

Teorem 4.3 ün bir uygulaması olarak aşağıdaki teoremi verelim.

\subsection{Teorem}

$(X, M, *)$ G-tam fuzzy metrik uzay, $\phi$ uzaklığ değiştiren fonksiyon ve $\psi:[0, \infty) \rightarrow[0, \infty), \psi(t)=0 \Leftrightarrow t=$ 0 özelliğini sağlayan alt yarı sürekli bir fonksiyon ve $\cup_{x \in X}[F x]_{\alpha} \subseteq g(X)$ olmak üzere $g: X \rightarrow X$ ve $F: X \rightarrow$ $W_{\alpha}(X)$ fuzzy fonksiyonu; her $x, y \in X$ ve her $t>0$ için

$\phi\left(\frac{1}{H_{M_{\alpha}}(F x, F y, t)}-1\right) \leq \phi\left(\frac{1}{M(g x, g y, t)}-1\right)-\psi\left(\frac{1}{M(g x, g y, t)}-1\right)$

şartını sağlasın. Bu durumda $g$ ile $F$ nin çakışık sabit fuzzy noktası vardır. Eğer, en az bir $x_{\alpha} \in C_{\alpha}(g, F)$ için $g^{2} x=g x$ ise $g$ ile $F$ nin ortak sabit fuzzy noktası vardır.

\section{İspat}

Önerme $4.1 \operatorname{den} g(E)=g(X)$ ve $g: E \rightarrow X$ bire bir olacak şekilde bir $E \subset X$ vardır.

$\mathcal{A}: g(E) \rightarrow W_{\alpha}(X), \mathcal{A}(g x)=F x$

şeklinde tanımlanan $\mathcal{A}$ dönüşümü, $g$ bire bir olduğundan iyi tanımlıdır.

$$
\begin{aligned}
\phi\left(\frac{1}{H_{M_{\alpha}}(\mathcal{A}(g x), \mathcal{A}(g y), t)}-1\right) & =\phi\left(\frac{1}{H_{M_{\alpha}}(F x, F y, t)}-1\right) \\
& \leq \phi\left(\frac{1}{M(g x, g y, t)}-1\right)-\psi\left(\frac{1}{M(g x, g y, t)}-1\right)
\end{aligned}
$$


elde edilir ki bu da $\mathcal{A}$ dönüşümünün, (14) eşitsizliğini ve Teorem 4.3 deki tüm şartları sağladığını gösterir. $\mathrm{O}$ halde $\mathcal{A}$ dönüşümü, Teorem 4.3 e uygulanırsa $\mathcal{A}, r \in[\mathcal{A}(r)]_{\alpha}$ olacak şekilde $r \in g(E)$ sabit fuzzy noktaya sahiptir. $\bigcup_{x \in X}[F x]_{\alpha} \subset g(X)$ olduğundan $\left(g r^{\prime}\right)_{\alpha}=r_{\alpha}$ olacak şekilde $r^{\prime} \in X$ vardır. Dolayısıyla $\left(g r^{\prime}\right)_{\alpha} \subset x_{n} \mathcal{A}\left(g r^{\prime}\right)=F r^{\prime}$ yani $g r^{\prime} \in\left[F r^{\prime}\right]_{\alpha}$ dır. Öyleyse $r_{\alpha}^{\prime}$ noktası, $F$ ile $g$ nin çakışık sabit fuzzy noktasidir. Yani $C_{\alpha}(g, F) \neq \emptyset$ dir.

Hipotezden, en az bir $x_{\alpha} \in C_{\alpha}(g, F)$ için $g^{2} x=g x$ olsun. $g^{2} x=g x \in[F x]_{\alpha}$ olduğundan $(g x)_{\alpha} F$ ile $g$ nin ortak sabit fuzzy noktasıdır.

\section{Tartışma ve Sonuçlar}

$\mathrm{Bu}$ çalışmada, Hausdorff fuzzy metrik uzaylarda uzaklığı değiştiren fonksiyonlar yardımı ile sabit ve ortak sabit fuzzy nokta teoremleri verilmiştir. Son yıllarda yapılan pek çok çalışma sayesinde bu alanın önemi giderek artmakta ve uygulama açısından yeni imkanlar sunmaktadır. Burada elde edilen sonuçlar, daha sonra yapılacak olan farklı büzülme tipleri ve farklı koşullar altında sabit fuzzy nokta araştırmaları için kullanılabilir.

\section{Teşekkür}

Makalenin inceleme ve değerlendirme aşamasında yapmış oldukları titiz ve özverili çalışmaları için sayın editörlere ve değerli katkılarından dolayı sayın hakemlere teşekkür ederim.

\section{Kaynaklar}

Abbas, M., Ali, B. and Vetro, C., 2015. Fixed Fuzzy Points of Fuzzy Mappings in Hausdorff Fuzzy Metric Spaces with Application. Iranian Journal of Fuzzy Systems, 12(3), 31-45.

Alaca, C., 2009. On Fixed Point Theorems in Intuitionistic Fuzzy Metric Spaces. Communications of the Korean Mathematical Society, 24(4), 565-579.

Ali, B. and Abbas, M., 2013. Suzuki Type Fixed Point Theorem for Fuzzy Mappings in Ordered Metric Spaces. Fixed Point Theory and Applications, 2013:19, 1-19.

Chang, C. L., 1968. Fuzzy Topological Space. Journal of Mathematical Analysis and Applications, 24, 182-190.

Chitra, A. and Subrahmanyam P. V., 1987. Fuzzy Sets and Fixed Points. Journal of Mathematical Analysis and Applications, 124, 584-590.

Dosenovic, T., Rakic, D., Brdar, M., 2014. Fixed Point Theorems in Fuzzy Metric Spaces Using Altering Distance. Filomat, 28(7), 1517-1524.

Estruch, V. D. and Vidal, A., 2001. A Note on Fixed Fuzzy Points for Fuzzy Mappings. Rendiconti
dell'Istituto di Matematica dell'Universita di Trieste, 32, 39-45.

George, A. and Veeramani, P.,1994. On Some Results in Fuzzy Metric Spaces. Fuzzy Sets and Systems, 64, 395-399.

Grabiec, M., 1988. Fixed Points in Fuzzy Metric Spaces. Fuzzy Sets and Systems, 27, 385-389.

Gregori, V., Morillas, S. and Sapena, A., 2011. Examples of Fuzzy Metrics and Applications. Fuzzy Sets and Systems, 170, 95-111.

Haghi, R. H., Rezapour, Sh.and Shahzad, N., 2011. Some Fixed Point Generalizations are not Real Generalizations. Nonlinear Analysis, 74(5), 1799-1803.

Heilpern, S., 1981. Fuzzy Mappings and Fixed Point Theorem. Journal of Mathematical Analysis and Applications, 83(2), 566-569.

Khan, M.S., Swaleh, M. and Sessa, S., 1984. Fixed Point Theorems by Altering Distances Between the Points. Bulletin of the Australian Mathematical Society, 30(1), 1-9.

Kramosil, O. and Michalek, J., 1975. Fuzzy Metric and Statistical Metric Spaces. Kybernetica, 11, 326334.

Lowen, R., 1976. Fuzzy Topological Spaces and Fuzzy Compactness. Journal of Mathematical Analysis and Applications, 56, 621-633.

Mihet, D., 2004. A Banach Contraction Theorem in Fuzzy Metric Spaces. Fuzzy Sets and Systems, 144, 431-439.

Nadler, S. B., 1969. Multivalued Contraction Mappings. Pacific Journal of Mathematics, 30(2), 475-488.

Naidu, S. V. R., 2003. Some Fixed Point Theorems in Metric Spaces by Altering Distances. Czechoslovak Mathematical Journal, 53(128), 205-212.

Nashine H. K. and Aydi, H., 2013. Coupled Fixed Point Theorems for Conctractions Involving Altering Distances in Ordered Metric Spaces. Mathematical Sciences, 7(20), 2013. 
Pao-Ming, P. and Ying-Ming, L., 1980. Fuzzy Topology: II. Product and Quotient Spaces. Journal of Mathematical Analysis and Applications, 77, 20-37.

Phiangsungnoen, S., Sintunavarat, W. and Kumam, P., 2014. Fuzzy Fixed Point Theorems in Hausdorff Fuzzy Metric Spaces. Journal of Inequalities and Applications, 2014(201), 1-10.

Popa, V. and Mocanu, M., 2009. Altering Distance and Common Fixed Points Under Implicit Relations. Hacettepe Journal of Mathematics and Statistics, 38(3), 329-337.

Rhoades, B. E., 2001. Some Theorems on Weakly Contractive Maps. Nonlinear Analysis: Theory, Methods and Applications, 47(4), 2683-2693.

Rodriguez-Lopez, J. and Romaguera, S., 2004. The Hausdorff Fuzzy Metric on Compact Sets. Fuzzy Sets and Systems, 147, 273-283.
Schweizer, B. and Sklar, A., 1960. Statistical Metric Spaces. Pacific Journal of Mathematics, 10, 314-334.

Shen, Y., Qiu, D., Chen, W., 2012. Fixed Point Theorems in Fuzzy Metric Spaces. Applied Mathematics Letters, 25, 138-141.

Türkoğlu, D., Alaca, C., Cho, Y. J., Yıldız, C., 2006. Common Fixed Point Theorems in Intuitionistic Fuzzy Metric Spaces. Journal of Applied Mathematics and Computing, 22, 411-424.

Veeramani, P., 2001. Best Approximation in Fuzzy Metric Spaces. Journal of Fuzzy Mathematics, 9, 75-80.

Wong, C. K., 1974. Fuzzy Topology: Product and Quotient Theorems. Journal of Mathematical Analysis and Applications, 45, 512-521.

Zadeh, L. A., 1965. Fuzzy Sets. Information and Control, 8, 338-353. 